\title{
Pressure aberrations inside the spinal canal during rear-end impact
}

\author{
K-U Schmitt ${ }^{1,2}$, M Muser $^{2}$, P Niederer ${ }^{1}$, F Walz ${ }^{2}$
}

\begin{abstract}
Minor soft tissue injuries of the cervical spine increasingly pose problems in public health. Such injuries are conveyed particularly often in rear-end automobile collisions at low impact speeds and it has been established that they may be associated with long-term impairment. As a possible cause for this type of injury it has been hypothesized that pressure pulses induced in cervical fluid compartments during the impact could damage the membrane of spinal nerve cells. To date, animal as well as cadaver experiments performed support this hypothesis.

A theoretical analysis has been undertaken in order to investigate the pressure and flow pulse emerging in a cervical fluid compartment under conditions representing rear-end impacts with a $\Delta \mathrm{v}$ of $15 \mathrm{~km} / \mathrm{h}$. Using the finite element (FE) method, a three-dimensional model of
\end{abstract}

\section{Introduction}

Traffic accident statistics as well as information from insurance companies show the increasing importance of cervical spine injuries (e.g., Temming And Zobel 1998, Hell et Al. 2000). In particular, soft tissue neck injuries reported frorm low-speed rear-end collisions are of continued concem in road traffic. Although these injuries - often called "whiplash" injuries - are usually classified as minor injuries (AIS1) and often do not exhibit a morphological manifestation, their high incidence rate and possible long-term consequences lead to signiticant societal costs (KRAFFT ET AL. 2000). Consequently, the biomechanics of these neck disorders has become a major issue in traffic safety.

The mechanisms that may cause these injuries are multiple and not all of them are yet biomechanically fully understood. However, several possible mechanisms have been identified: a shearing movement between the vertebrae has, for instance, been associated with lesions of intervertebral joint facets (YANG ET AL. 1997). Other studies hypothesise that soft tissue neck injuries are caused by hyperextension of the neck, maximum neck moments and head excursion angles (MERTZ AND PATRICK 1971, WAlZ AND MUSER 1995, 2000). A further possibility proposed by ALDMAN ET AL. (1986) takes into consideration a pressure gradient that develops in the venous and cerebrospinal fluid of the spinal canal. It was predicted that transient pressure changes would occur in the central nervous system during the swift extension-flexion motion of the human cervical spine. The kinematics underlying this motion has been identified as the so-called S-shape which develops in the early stage of a rear-end impact. During this type of movement, the spinal canal alters its length (BREIG, 1960) and thus its inner volume, the cervical spine was developed. The model consists of eight vertebrae (C1-T1), the intervertebral discs, the intervertebral joints, all the major ligaments, most of the neck muscles and the head. Additionally, a typical venous blood vessel was included. To determine the pressure behaviour inside the blood vessel, fluid-structure interaction was taken into account.

For the time interval including the development of the S-shape, the pressure pulses were calculated and found to be in qualitative agreement with the reported measurements. The shear stresses acting on the vessel wall can be determined from the associated flow pulses. An extrapolation of the results into the interstitial space where nerve cells are located at this stage does not allow assessment of whether a damage threshold may be reached.

inducing alterations in blood volume. Assuming that all tissues and fluids inside the spinal canal are incompressible, the change of volume as seen in extension-flexion motions has to be compensated by fluid transportation. The flow of the cerebrospinal fluid is regarded to be of minor importance in the time scale of a swift neck motion because of its greater flow resistance in the subarachnoidal space and its inertia dominated flow behaviour (LOTH ET AL. 2001). Hence, the compensation of the volumetric changes is postulated to be solely achieved by blood flow. Considering that, generally, the capacity of the venous system is much higher than that of the arterial system, the fact that there are no valves associated with these venous vessels, and taking into account that the internal and external vein plexuses are connected via bridging veins, the blood can easily move within the plexuses as well as in between the plexuses. Therefore, the venous blood which is forced to flow along the plexus venosus vertebralis and its bridging veins will be subjected to rapid changes in its velocity, which in turn will produce pressure gradients. Such pressure gradients can then be expected to increase the shear stress to the cervical soft tissue.

Under normal physiological conditions, there is no wave propagation in the venous system. Nevertheless, providing that the pressure amplitude and the steepness of the wave front are sufficiently high, shock-like transitions are conceivable. It has been argued that this effect, which sometimes is referred to as the "water hammer" effect, may be one of the reasons for the injury (BOSTRÖM ET AL. 1996).

Based on this theory, experiments using pigs were performed (SVENSSON ET AL. 1993, 2000) showing that the pressure amplitude recorded at the $\mathrm{C} 4$ level experienced a significant aberration. Furthermore, a histopathologic investi-

${ }^{1}$ Institute of Biomedical Engineering, University and Swiss Federal Institute of Technology (ETH), Zurich; ${ }^{2}$ Working Group on Accident Mechanics, University and Swiss Federal Institute of Technology (ETH), Zurich

Correspondence: Dr Kai-Uwe Schmitt, Institute of Biomedical Engineering D-ITET, University and Swiss Federal Institute of Technology (ETH),

Gloriastrasse 35, ETH Zentrum ETZ K 64, Zurich, Switzerland. Telephone +41-1-632-75-36, e-mail schmitt@biomed.ee.ethz.ch 
gation correlated these findings with the damage of the cell membranes of the spinal ganglia (ÖRTENGREN ET AL. 1996). Consequently, taking into account these investigations as weIl as the theoretical aspects mentioned above, a hydrodynamic model was developed by BOsTRÖM ET AL. (1996) resulting in the proposal of the neck injury criterion NIC.

In addition, PMHS (post mortem human subject) tests have been performed (EICHBERGER ET AL. 2000) where the pressure effects in the spinal canal during rear-end impacts were measured. The findings from this study revealed a similar pressure pattern to those obtained from the animal experiments. A correlation between the pressure amplitude and the NIC was also found.

Focusing on cell damage, the flow of fluid over cells gives rise to potential concomitant stimuli, including hydrostatic pressure gradients and shear stress. Several studies have analyzed this shear stress phenomenon (e.g., GAN ET AL. 1999, THibAult 1993, BORN ET AL. 1992, KRETZMER ET AL. 1991) but few deal with traumatic injury models (MORRISON ET AL. 1998, LaPlaca ET AL. 1997, Ellis ET AL. 1995). Shear levels at which damage was observed have been reported, but the values found depend strongly on the experimental setup, the cell type used as well as on the duration for which the cells were exposed to shear forces. However, the results show that cells are generally susceptible to shear, in particular with respect to membrane properties such as membrane permeability and thus the damage leads to loss of viability. Hereby different dyes and lactate dehydrogenase ( $\mathrm{LDH})$ release were used to analyze the extent of cell injury (ELLIS ET AL. 1995, LAPLACA ET AL. 1997). Shear stresses reported in the literature as causing damage vary considerably. Regarding the ability of injured cells to repair or replace damaged cell structures, it was found that cells recover at a rate which is dependent on the degree of injury (ELLIS ET AL. 1995). However, in such studies, repair occurred while the cells were kept in a normal cell culture medium. Injured cells placed in a less enriched medium or subjected to a secondary insult, such as hypoxia, may not recover as weIl. With respect to the findings by ÖRTENGEN ET AL., it has to be pointed out that these represent solely the situation shortly after the experiments and does not account for any secondary effects, consequently, possible long-term impairment can not be assessed from these experiments.

The aim of this study was to develop a theoretical model that allows for the analysis of the pressure phenomena occurring during the extension-flexion motion of the neck induced by a rear-end impact. For this purpose, the kinematics of the solid neck structures, obtained from a motion simulation, have to be combined with a fluid model. Of particular interest is the fluid-solid interaction developed during the critical S-shape of the cervical spine.

Approaches of this kind have been discussed in computational fluid dynamics (CFD) (e.g., Hoogstraten ET AL. 1996, Lynch et Al. 1996, PerkTold and Rappitsch 1995), where techniques have emerged enabling the study of hemodynamics in physiological geometries, for example, the coronary arteries or arterial bifurcations. The computational solutions yield velocities and pressures at all points within the fluid compartment and can provide information on wall shear stresses at the surfaces.
In a recent study, the combination of CFD and CSD (computational structure dynamics) was investigated, i.e., a deformation of the fluid compartment was also taken into account. Problems addressing such fluid-structure interactions dealt with, for instance, the behaviour of collapsible tubes (HeIL 1998) or the blood flow inside coronary arteries on the pumping heart (SANTAMARINA ET AL. 1998).

The study presented here introduces the technique of CFD/CSD coupling based on the finite element (FE) method to trauma-biomechanics. An FE model of the cervical spine was combined with an FE model of a cervical blood vessel to determine the effects on the blood flow during rear end impact.

\section{METHODOLOGY}

A theoretical approach using the Finite Element (FE) technique that allows analysis of the flow of blood inside a characteristic vessel undergoing the critical S-shape deformation was developed. The FE model in this study was a complex model of the cervical spine which can, for practical purposes, be thought of as consisting of two separate parts: a solid model and a fluid model.

The basis for the solid model was a three-dimensional FE model of the cervical spine and the first thoracic vertebra developed previously (YANG ET AL. 1998). The geometry of the model was derived using MRI scans from a male volunteer. Therefore, it exhibits a high anatomical accuracy; however, it represents an individual case. The volunteer (height: $1.74 \mathrm{~m}$, weight: $75 \mathrm{~kg}$ ) was chosen in order to replicate a 50 th percentile Hybrid III dummy as closely as possible.

As shown in Figure 1, the model consists of eight vertebrae (Cl-Tl), the intervertebral discs, the intervertebral joints, all major ligaments and the head. Most of the neck muscles believed to be relevant in rear-end collisions were also included in the model. The muscles were implemented as bar elements using a Hill-type formulation and thus take into account active and passive muscle characteristics. The points of origin of the neck muscles were adopted from the literature (DENG AND GOLDSMITH 1987, LANG 1991). In order to simulate the curving of long extensor muscles around the vertebrae during neck bending some muscles (e.g., M. longus capitis) were modelled by chains of connected Hill-type bars. The intermediate nodes of these chains were attached to the respective vertebrae. A reflex time of $50 \mathrm{~ms}$ was used for all muscles according to EMG measurements (HeLl ET AL. 1999); other muscle parameters such as weight, volume, force-length characteristics, and force-velocity characteristics were taken from the literature (WITTEK ET AL. 1998).

Additionally, the outer bounds of a fluid compartment situated inside the spinal canal were added to the model. Shell elements were used to model the walls of the fluid compartment whose dimensions represent a typical blood vessel of the plexus venosus vertebralis with a diameter of $2.5 \mathrm{~mm}$ and a length of $118 \mathrm{~mm}$ (Figure 1). To connect the vessel to the spine model, it was attached to the posterior side of the ligamentum longitudinale posterior. Thus the vessel was deformed according to this ligament. For the 530 shell elements that were assumed to be elastic, homogenous and isotropic, mechanical properties were chosen to represent a typical venous wall. Ring-shaped rigid body definitions were introduced to stabilize the vessel and to prevent the cross section from collapsing. For the modified sol- 


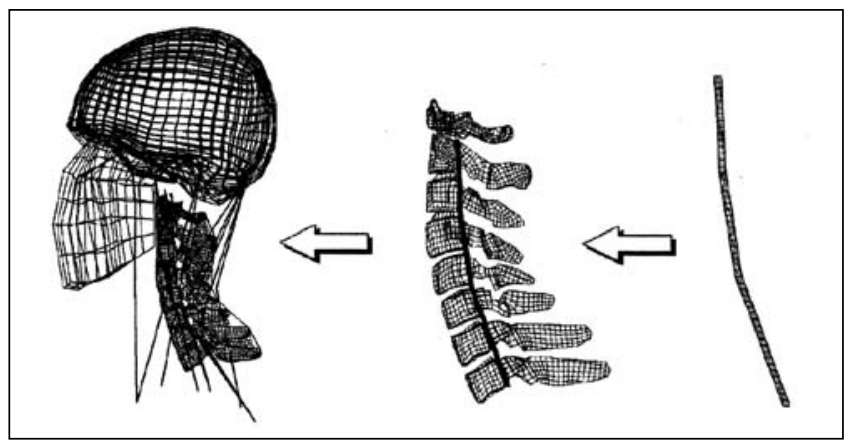

Figure 1) 3D FE model of the cervical spine including a venous blood vessel situated in the spinal canal

id model, validation on the basis of volunteer sled test data obtained from Japan Automotive Research Institute was performed. The FE model agreed well with the data (LINDER, SCHMitT ET AL. 2000). Due to the lack of data for comparison, fluid flow could not be taken into account in this validation process. However, the fluid flow model itself was validated for specific, though simpler, fluid dynamic problems resulting in favourable agreement (ESI 1998, NIEDERER ET AL. 2001).

In order to take into account the fluid structure interaction, the fluid filled volume inside the venous blood vessel was modelled separately. The volume was automatically meshed by 5364 tetrahedral elements. An initial flow velocity of $0.2 \mathrm{~m} / \mathrm{s}$ in the vertical direction from cranial to caudal was assumed. The inlet velocity profile was flat (plug flow) and steady. The fluid representing blood was assumed to be incompressible, homogeneous, and newtonian. Material parameters describing the fluid were taken from the literature (cf. e.g., BOSTRÖM ET AL. 1996) to be $0.0035 \mathrm{~kg} / \mathrm{m} \cdot \mathrm{s}$ for the viscosity and $1050 \mathrm{~kg} / \mathrm{m}^{3}$ for the density. For the entire calculation, laminar flow was assumed.

The boundary conditions included no slip at the tube wall, (i.e., the fluid velocity at the tube wall equals tube wall velocity). A far field condition by means of a constant pressure condition was applied at the outlet. In the interpretation of the results, the regions of the tube near the entrance and near the exit were not included.

For the FE calculations the following procedure was used: prior to the coupled fluid structure analysis, a steady state solution for the fluid model was gained (i.e., a CFD calculation was performed without moving the fluid compartment). This step was included to ensure a fully developed and uniform flow field inside the vessel at the beginning of the fluid structure interaction computation.

Then the CFD-CSD calculation was started and the solid model was subjected to a crash pulse, resulting in the deformation of the model, thus, allowing the recording of the deformation of the fluid compartment. In the next step this deformation was imposed to the fluid model and the interaction with the fluid herein was calculated. Finally, the reaction of the fluid, in terms of load on the vessel walls, was taken as a boundary condition for the solid model. Figure 2 illustrates this iteractive approach.

The crash pulse used in the deformation process represents a pulse with a peak acceleration of $4 \mathrm{~g}\left(=39.2 \mathrm{~m} / \mathrm{s}^{2}\right)$ as described in the PMSH test by EICHBERGER ET AL. The translational crash pulse leading to a $\Delta \mathrm{v}$ of approximately $15 \mathrm{~km} / \mathrm{h}$

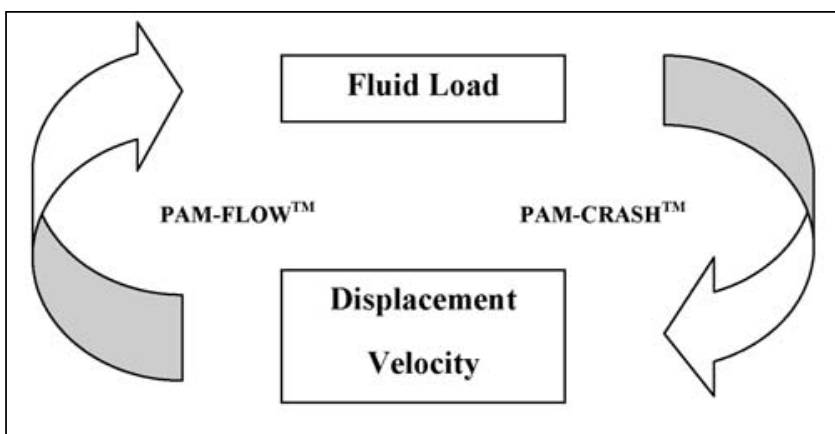

Figure 2) Calculation scheme underlying the software tool used for the coupling approach

was applied to the TI vertebra in the $\mathrm{x}$-direction (referring to SAE 1211/1 sign convention).

All calculations were carried out with the FE program package PAM (ESI 1998) of which the PAM-CRASH ${ }^{\mathrm{TM}}$ module dealt with the structural mechanics while the PAM-FLOW ${ }^{\mathrm{TM}}$ module was used for the fluid dynamics (Figure 2). The scheme underlying the coupling of these two modules is based on a socalled weak formulation. Hence, the FE equations for the structure and the fluid were solved separately, whereby two different time steps, depending on the momentary situation, are chosen. To cope with this formulation, a fractional step algorithm was implemented. An ALE (arbitrary Lagrangian Eulenan) formulation was implemented and as for the large displacements of the spine model and in particular of the vessel walls, the use of Lagrangian co-ordinates was advantageous, whereas the fluid equations were based on an Eulerian description. Consequently, the fluid mesh was adjusted by means of an automatic mesh generator as the vessel wall deformed.

To analyze the pressure inside the blood vessel, the velocity flow fields and the shear stress on the vessel wall were determined as functions of time. Due to the volumetric remeshing of the fluid mesh, the results were evaluated at a fixed number of time history points located in the sagittal plane. All calculations were performed on an IBM 397 POWER2 SuperChip workstation.

Following the same scheme, additional calculations were carried out varying the vessel geometry. Beside a smaller vessel with a diameter changed to $2 \mathrm{~mm}$, an ellipsoidal cross section with a short semiaxis of $0.9375 \mathrm{~mm}$ (in the sagittal direction) and a long semiaxis of $1.6665 \mathrm{~mm}$ (lateral axis) was chosen. Thus, the cross sectional area of the ellipsoidal vessel equals that of the vessel with a diameter of $2.5 \mathrm{~mm}$. Results obtained from these variations were used to check for the stability and comparability of the method under consideration.

\section{RESULTS}

The entire simulation covered a time interval of $65 \mathrm{~ms}$. This interval was chosen such that the development of the S-shape was included (see sequence shown in Figure 3).

The pressure distribution inside the vessel was determined as a function of time. The reported pressure values represent the results at the centre of the cross section. Due to the continuous remeshing, it was not possible to ensure that for all time steps, suitable nodes were exactly at the mid cross section, in such cases, time history points that were as close as possible 


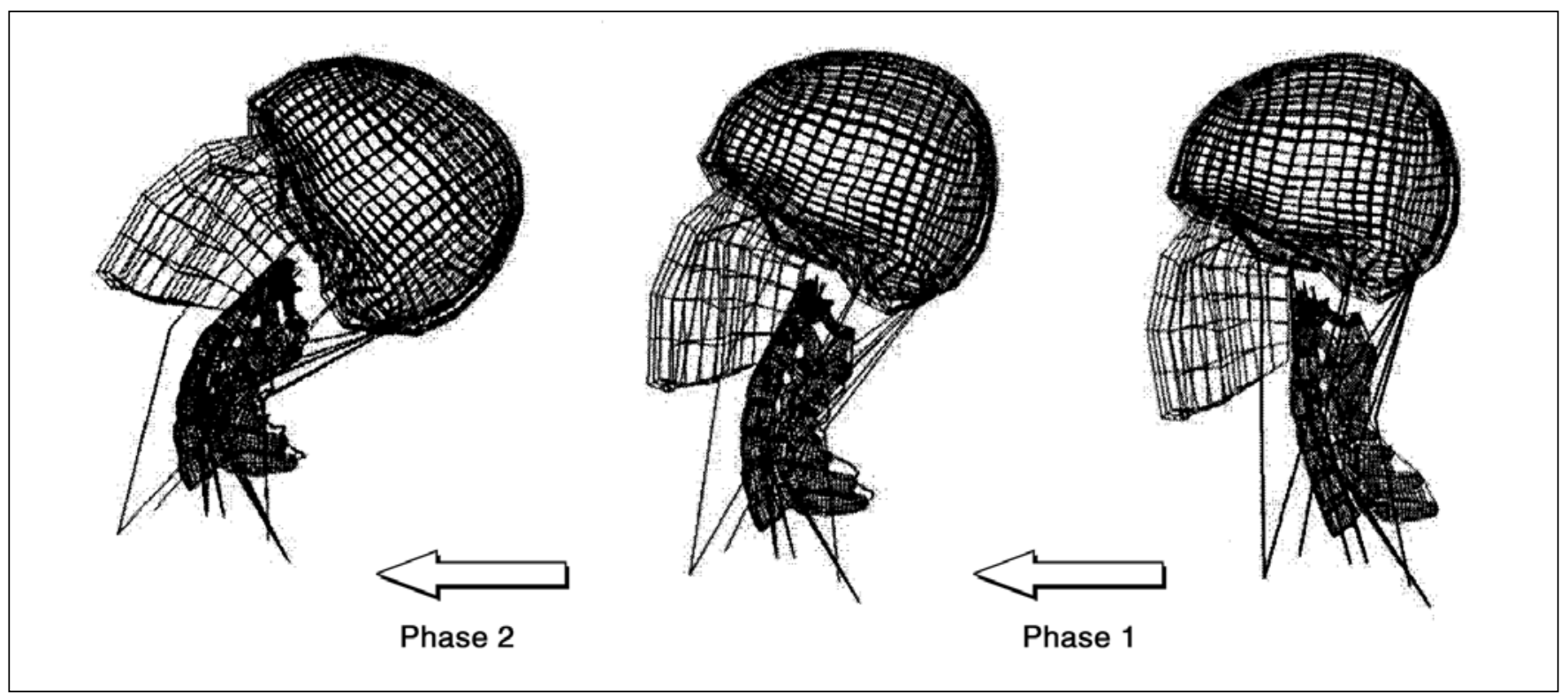

Figure 3) FE model showing the initial configuration at $0 \mathrm{~ms}$ (right), S-shape deformation at $80 \mathrm{~ms}$ (middle) and extension motion at $120 \mathrm{~ms}$ (left)

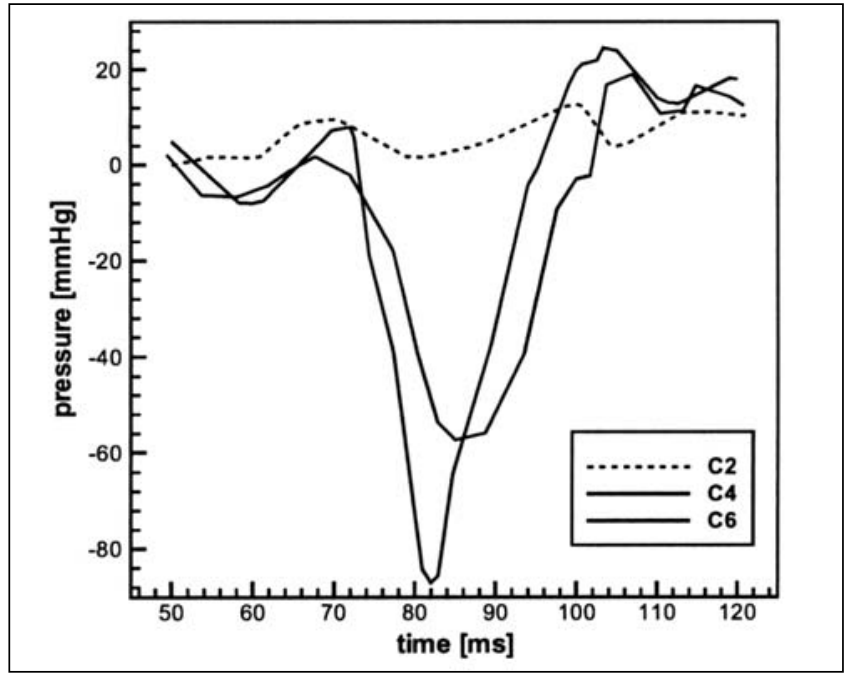

Figure 4) Pressure computed at the C2, C4 and C6 levels. The calculation of the fluid structure interaction was started at time $=50 \mathrm{~ms}$ of the total FR stimulation. The vertical axis gives the pressure $(1 \mathrm{mmHg}=0.133 \mathrm{kPa})$

to it were taken instead. Since the pressure exhibits small variations over the cross section only in the case of a primarily one-dimensional flow, this aspect is of minor significance. The pressure results were filtered numerically using a CFC 1000 filter according to the experiments by EICHBERGER ET AL. (2000). The pressure was analyzed at three different levels of the cervical spine: at the $\mathrm{C} 2, \mathrm{C} 4$ and $\mathrm{C} 6$ levels. The results are shown in Figure 4. For both the C4 and C6 levels, a significant drop in the amplitude followed by an increase was observed in the interval calculated. The negative peak of the pressure coincided with the maximal S-shape deformation of the spine model. For the C4 level, an amplitude of $87 \mathrm{mmHg}(=11.571 \mathrm{kPa})$ was obtained, while for the C6 level, the amplitude was about

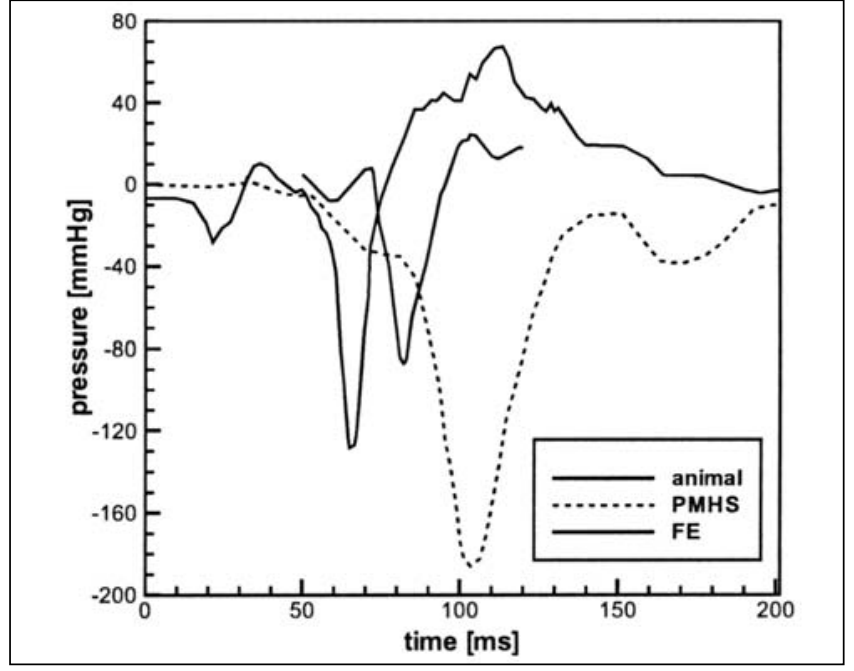

Figure 5) The comparison of the pressure results at the C4 level with results from the experiments is shown. For the animal experiments, the results presented in SVENSSON ET AL. (2000) for an extension motion at a pull force of $600 \mathrm{~N}$ without head restraints at the $\mathrm{C} 4$ level were chosen. The results for the PMHS experiments shown are taken from EICHBERGER ET AL. (2000) for test P98_09

35\% smaller. At the C2 level, no characteristic pressure peak was determined.

For comparison, representative curves taken from animal experiments by SVENSSON ET AL. (2000), PMHS experiments by EICHBERGER ET AL. (2000) and results at the C4 level are shown in Figure 5.

In addition, the flow pattern within the vessel was analyzed. A plug profile was used as the initial condition. This type of velocity profile prevailed in the beginning of the deformation phase. However, as the deformation reaches the maximum $\mathrm{S}$-shape, a change in the flow direction occurring at the same time as the peak of the pressure amplitude can be seen in the part of the vessel cranial to the $\mathrm{C} 4$ level. Figure 6 illustrates the 


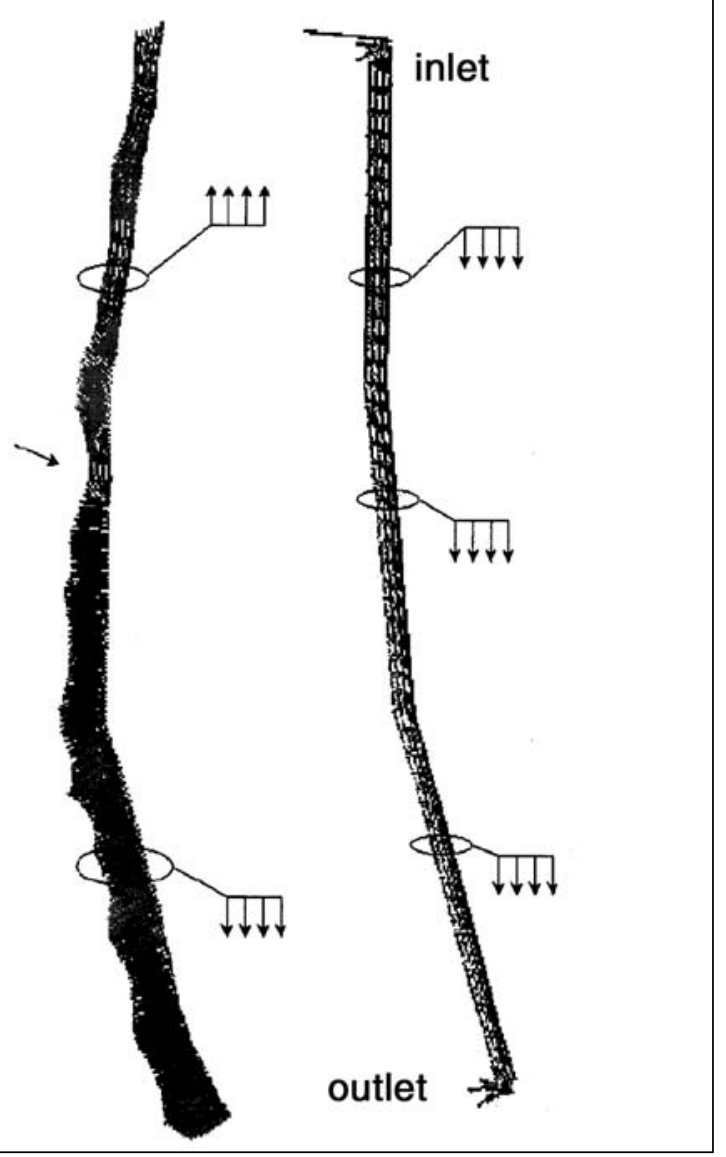

Figure 6) Resultant velocity inside the vessel at the beginning of the fluid structure interaction (right) and at time $=75 \mathrm{~ms}$ (left). The change of direction in the part above the $\mathrm{C} 4$ level can be seen; the arrow points on the area where the velocity is very small and not oriented in one particular direction

flow directions at different times. The shear stress on the vessel wall was also computed. Maximal values in the order of $0.76 \mathrm{~N} / \mathrm{m}^{2}$ have been calculated and were mainly situated at the anterior side of the vessel. Varying the diameter of the vessel lead to, qualitatively, the same results. Whereas for the ellipsoidal geometry, only minor differences in the results were found (amplitudes varying less than 5\%) and the maximal shear stress for the smaller diameter was determined to be $0.62 \mathrm{~N} / \mathrm{m}^{2}$. The pressure behaviour in the latter case is shown in Figure 7.

\section{DISCUSSION}

Based on the FE method, a novel technique that takes into account fluid structure interaction was applied. To the best of our knowledge, such a technique has not yet been used in trauma biomechanics. With this method at hand, it was possible to analyze the fluid behaviour inside a venous blood vessel during a simulated rear-end impact. The results from this study are intended to provide further understanding of the underlying mechanism of soft tissue neck injuries.

The computed flow pattern clearly indicates a marked change in the blood flow as a result of the impact. Simultaneously, a significant drop in pressure during the first phase of the motion sequence associated with a typical whiplash resulting from a rear-end impact is observed. Hence, the calculations strongly suggest that a similar effect as described in the literature is likely to occur under low speed rear-end impact conditions, although the slope of the pressure amplitude is far from a shock-like transition ("water hammer"). Consequently, these findings support, at least to some extent, the hypotheses first mentioned by ALDMAN ET AL. (1986).

The determined pressure amplitude varied for different levels: at the $\mathrm{C} 4$ and $\mathrm{C} 6$ levels a distinct drop followed by an increase was calculated. This result is qualitatively in agreement with the measurements by SVENSSON ET AL. (1993) and EICHBERGER ET AL. (2000). The magnitude computed is slightly lower compared to the experimental results given, though it is closer to that of the animal experiments. Considering the fact that the fluid is incompressible, those differences can be attributed to differences in the anatomy modelled, and thus, are due to the details of the shape changes of the fluid volume during deformation. Furthermore, the experiments, in particular those performed with the PMHS, exhibit a large variability in the measurements. Because the anatomical data are not available, the FE model could not be designed to simulate the experiments with which it is compared. The essentially one-dimensional flow pattern also has to be considered a simplification of the highly tortuous venous plexus. Thus, the time at which the S-shape developed is especially different. These results as well as those with different timing for the pressure drop, can be seen in Figure 5. Uncertainties regarding the constitutive properties assumed in the model, particularly in terms of fluid flow boundary conditions, are inherent in all calculations. However, those assumptions are also used in other studies in the field of fluid structure interaction (e.g., SANTAMARINA ET AL. 1998). Further limitations are concerned with the input data. As no other data other than the $\mathrm{Tl} \mathrm{x}$-acceleration data were available from the cadaver experiments, axial and rotational movement was neglected. This might also contribute to the differences in the responses shown in Figure 5.

For the pressure results at the $\mathrm{C} 2$ level, no characteristic pressure aberration was found. The amplitude was relatively small; a fact that can also be found in the experimental results.

Analysis of the shear stresses at the vessel walls revealed maximal values in the order of $0.76 \mathrm{~N} / \mathrm{m}^{2}$. This value seems to be small. Generally, for arteries it is reported that the axial shear stress in flowing blood at the endothelial surface is in the order of 1 to $2 \mathrm{~N} / \mathrm{m}^{2}$ [FuNG 1993]. For veins, the shear stress of the flow acting on the vessel walls is smaller because the diameter is larger than that of arteries of the same generation, but the volume flow rate is similar. Hence, the value calculated in this study might possibly even exceed physiological values for the veins of the plexus of interest. A number of laboratory experiments has been performed on cell cultures in order to determine the influence of a controlled mechanical loading on the properties of cellular membranes. The loading conditions chosen were intended to mimic traumatic situations extrapolated from macroscopic correlates. An overview of the published tests performed with various types of nerve cells in vitro is given in MORRISON ET AL. (1998). The general concept underlying most of these experiments is that it is ultimately the extent of mechanical deformation of the cellular membrane which determines the level of injury. Accordingly, it has usually been attempted to determine cellular deformations. Loading conditions, including the target values applied in these test series, 

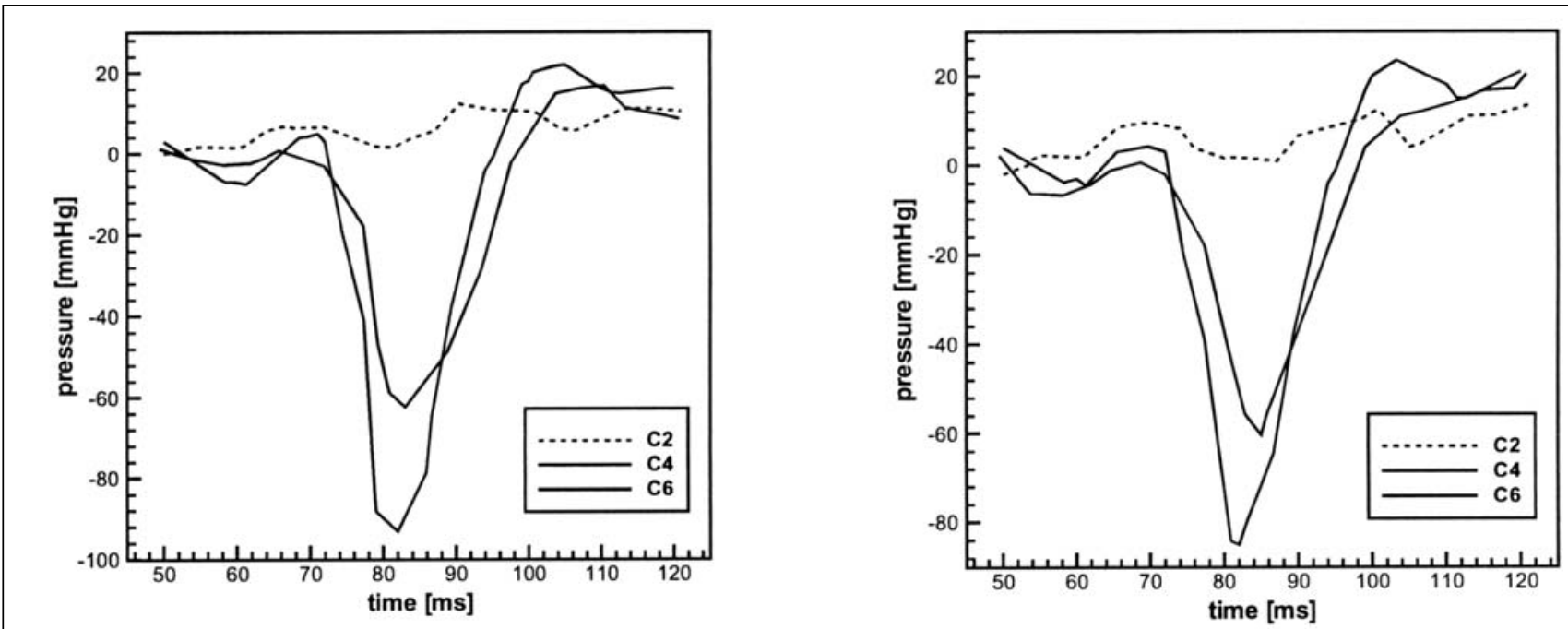

Figure 7) Pressure results for the variation of the vessel diameter: smaller but round cross-section (left) and ellipsoidal geometry (right)

considered to be crucial in view of injury production, differ considerably. A systematic criterion cannot be derived from these experiments at this time because it is difficult to relate the laboratory loading patterns to those prevailing in humans under realistic impact conditions. In cases where cellular damage results, secondary mechanisms (mostly boundary effects) have to be taken into account. Additional factors such as the loading rate might also be important for cell injury. Furthermore, recent research suggests that the shear level "seen" by the cell could be several-fold greater than that described by the macroscopic wall shear stress (HUNG ET AL. 2001).

The results of this study do not allow assessment of whether the staining observed by ÖRTENGREN ET AL. is solely due to shear stresses imposed by the S-shape deformation and associated pressure aberrations. In comparison to the rate of change and the amplitude of the shear stress calculated with cellular injury models found in the literature, it might weIl be within the range of potentially harmful shear stresses. BORN ET AL. (1992) for instance, reported a range of $19 \times 10^{-3} \mathrm{~N} / \mathrm{m}^{2}$ to $100 \mathrm{~N} / \mathrm{m}^{2}$ with varying exposure times. Yet, it has to be taken into account that the experimental setups mentioned differ from the situation in vivo. The spinal ganglia found to be injured in the animal experiments may be more vulnerable because of stress concentrations at the spinal cord outlet. Therefore, relatively low shear stress amplitudes emerging from the fluid phenomenon could possibly interact or be superimposed with shear stress induced by the kinematics of the motion. Other effects influencing cell membrane viability also need to be considered: the role of extracellular fluid flow in the vicinity of the nerve cells, for example, remains unknown. Furthermore, the failure mechanism leading to an increasing permeability of the cell membrane is unknown (i.e., whether membrane damage is caused mechanically and/or by changes of biological functions of the membrane). Bearing in mind that the results reported by ÖRTENGREN ET AL. represent the situation directly after impact, factors influencing the ability of the cell to recover or repair cannot be assessed. Consequently, no conclusion with regard to permanent impairment can be drawn.
Regarding the model which included a vessel with an ellipsoidal cross section, the results remained more or less constant with respect to the $2.5 \mathrm{~mm}$ round vessel. This fact might be attributed to the unchanged fluid volume and thus seems to suggest that the pressure aberrations are not influenced much by slight changes in geometry. Bearing in mind that the blood pressure is, generally speaking, more stable than flow (GUYTON 1996), this effect is not surprising.

A smaller vessel diameter resulted, as expected, in a smaller calculated shear stress, whereas the pressure did not vary much due to its relative stability as mentioned above.

\section{CONCLUSIONS}

In order to elucidate the injury mechanism of soft tissue cervical spine injuries under low speed impact conditions, the gross deformation of the neck and the interaction with the fluid inside a venous blood vessel were analyzed. A newly developed software tool was used for this coupling approach which has proven reasonable to calculate fluid structure interactions in accident mechanics. A major limitation of this study was the assumed fluid flow boundary conditions.

The results indicate a characteristic initial pressure drop at the C4 level of the vessel, followed by an increase when subjected to the S-shape deformation. Comparing the numerical results with those of the experiments, a similar phenomenon can be found. In addition, shear stresses on the vessel wall were computed. The values obtained do not indicate a clear coherence to the experimental findings of cell membrane damage. Nevertheless, they do not exclude this possibility, but further investigations on injury models of cells are necessary.

Bearing in mind that the neck experiences a similar type of inertial loading in low speed frontal collisions, a similar fluid structure interaction leading to, qualitatively, the same pressure phenomena seems possible.

ACKNOWLEDGEMENTS: The authors wish to thank Prof. K. Yang for his help. The valuable advice from Dr. D Vineteler, ESI Paris, is also gratefully acknowledged. 


\section{REFERENCES}

1. Aldman B. An analytical approach to the impact biomechanics of head and neck. Proc. 30th Annual AAAM Conference. 1986:439-54

2. Born C, Zhang Z, Al-Rubeai M, Thomas CR. Estimation of disruption of animal cells by laminar shear stress. Biotechnol Bioeng 1992;40:1004-10.

3. Boström O, Svensson MY, Aldman B, ET AL. A new neck injury criterion candidate - based on injury findings in the cervical spinal ganglia after experimental neck extension trauma. Proc. IRCOBI Conf. Dublin, 1996:123-36.

4. BREIG A. Biomechanics of the central nervous system. Stockholm: Almqvist \& Wiksell, 1960

5. Deng YC, Goldsmith W. Response of a human head/neck/uppertorso replica to dynamic loading - II. Analytical/numerical model. J Biomech 1987;20:487-97.

6. Eichberger A, Darok M, StefFan H, Leinzinger PE, Boström O, SvEnsSON MY. Pressure measurements in the spinal canal of postmortem human subjects during rear-end impact and correlation of results to the neck injury criterion. Accid Anal Prev 2000;32:251-60.

7. Ellis EF, McKinney JS, Willoughby KA, Liang S, Povlishock JT. A new model for rapid stretch-induced injury of cells in culture: Characterization of the model using astrocytes. J Neurotrauma 1995; $12: 325-39$.

8. ESI Paris. PAM-CRASH and PAM-FLOW User's Manuals. Paris:ESI, 1998.

9. FUNG YC. Biomechanics: Mechanical properties of living tissues, 2nd Edition. Springer Verlag, 1993.

10. GAN L, SJÖGREN LS, DOROUdi R, JEM S. A new computerized biomechanical perfusion model for ex vivo study of fluid mechanical forces in intact conduit vessels. J Vasc Res 1999;36:68-78.

11. Guyton A. Textbook of Mmedical Physiology. Philadelphia: WB Saunders, 1996.

12. Heil M. Stokes flow in an elastic tube - a large-displacement fluidstructure interaction problem. Intern J Numerical Methods in Fluids 1998;28:243-65.

13. Hell W, Langwieder K, Walz F, Kramer M, Hartwig E. Consequences for seat design due to rear end accident analysis, sled tests and possible test criteria for reducing cervical spine injuries after rear end collision. Proc. IRCOBI Conf. Sitge.1999:243-59.

14. Hell W, Schick S, LANGWIEDER K. Epidemiology of cervical spine injuries in rear-end collisions and influence of different anthropometric parameters in human volunteer tests. In: Yoganandan and Pintar, eds. Frontiers in Whiplash Trauma. Amsterdam: IOS Press, 2000

15. Hoogstraten HW, Kootstra JG, Hillen B, Krijger JKB, WENSING PJW. Numerical simulation of blood flow in an artery with two successive bends. J Biomechanics 1996;29:1075-83.

16. Hung C, COSTA K, Guo X. Fluid flow effects on bone cells: Influence of flow-cell substrate interactions and cell mechanical properties; Proc. 2001 Workshop on Fluid Flow in Bone. $<$ http://frangos.ucsd.edu>, 2001.

17. Krafft M, Kullgren A, Tingvall C, Boström O, Fredriksson R. How crash severity in rear impacts influences short- and long-term consequences to the neck. Accid Anal Prev 2000;32:187-95.

18. Kretzmer G, SchÜGERL K. Response of mammalian cells to shear stress, Appl Microbiol Biotechnol 1991;34:613-6.

19. LANG J. Klinische Anatomie der Halswirbelsäule; Georg Thieme Verlag: Stuttgart.
20. LaPlaca M, Thibault LE. An in vitro traumatic injury model to examine the response of neurons to a hydrodynamically-induced deformation. Ann Biomed Eng 1991;25:665-77.

21. Linder A, Schmitt K-U, Walz F, Ono K. Neck modeling for rearend impact simulations - a comparison between a multi body system (MBS) and a finite element (FE) model. Proc. IRCOBI Conf. Montpellier; 2000:491-4.

22. LyNCH DG, Walters SL, PedLey TJ. Flow in a tube with nonuniform, time-dependent curvature: Governing equations and simple examples. J Fluid Mechanics 1996;323:237-65.

23. Mertz HJ, PATRICK LM. Strength and response of the human neck. Proc. 15 th Stapp Car Crash Conf. New York, 1971:207-55.

24. Morrison B, SaAtman K, Meaney D, Mclntosh T. In vitro centra nervous system models of mechanically induced trauma: A review. J Neurotrauma 1998;15:911-28.

25. Niederer P, Schmitt K-U, Muser M, Walz F. The possible role of fluid/solid interactions in minor cervical spine injuries. Proc. ASME Bioengineering Conf. Snowbird, 2001.

26. ÖRTENGREN T, Hansson HA, LÖVsund P, Svensson MY, SUNESON A, SÄLJÖ A. Membrane leakage in spinal ganglion nerve cells induced by experimental whiplash extension motion: A study in pigs. J Neurotrauma 1996;13:171-80.

27. PerkTOld K, Rappitsch G. Computer simulation of local blood flow and vessel mechanics in a compliant carotid artery bifurcation mod61. J Biomech 1995;28:845-56.

28. Santamarina A, Weydahl E, Siegel JM Jr, Moore JE JR. Computational analysis of flow in a curved tube model of the coronary arteries: Effects of time-varying curvature Ann Biomed Eng 1998;26:944-54.

29. Svensson MY, Aldman B, Hansson HA, et al. Pressure effects in the spinal canal during whiplash extension motion: A possible cause of injury to the cervical spinal ganglia. Proc. IRCOBI Conf. Eindhoven, 1993:189-200.

30. Svensson MY, Boström 0, Davidsson J, ET AL. Neck injuries in car collisions - a review covering a possible injury mechanism and the development of a new rear-impact dummy. Accid Anal Prev 2000;32:167-75.

31. Temming J, Zobel R. Frequence and risk of cervical spine distortion injuries in passenger car accidents: Significance of human factors data. Proc. IRCOBI Conf. Göteborg, 1998:219-33.

32. Thibault LE. Isolated tissue and cellular biomechanics. In: Nahum and Melvin, editors. Accidental lnjury - Biomechanics and Prevention. Springer Verlag, 1993.

33. WALZ F, MuSER M. Biomechanical aspects of cervical spine injuries. SAE Intemational Congress and Exhibition. Detroit, Michigan; SAE 950658 in SP-1077, 1995.

34. WALZ F, MUSER M. Practical biomechanical aspects of soft tissue neck injuries from real-world low-speed collisions. In: Yoganandan and Pinter, eds. Frontiers in Whiplash Trauma. Amsterdam: IOS Press, 2000.

35. Wittek A, Kajzer J, Haug E. Application of new PAM-safe muscle model. PAM Users Conference in Asia, 1998:337-55.

36. Yang KH, Begeman PC, Muser M, Niederer P, Walz F. On the role of cervical facet joints in rear end impact neck injury mechanisms. SP-1226; SAE 970497; 1997:127-29.

37. Yang KH, Zhu F, LuAn F, ZhaO L, Begeman P. Development of a finite element model of the human neck. Proc. 42nd Stapp Car Crash Conf. Tempe; SAE-983157, 1998:195-205.

This material was presented at the International Congress on Whiplash Associated Disorders, Berne, Switzerland, March 8 to 10, 2001. The paper appeared originally in the book "Whiplash Associated Disorders" - medical, biomechanical and legal aspects, published by Staempfli Publishers Ltd, Berne 2002. The paper is published in North America in Pain Research $\mathbb{E}$ Management with the permission of Staempfli Publishers Ltd. 


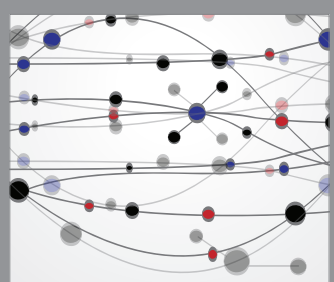

The Scientific World Journal
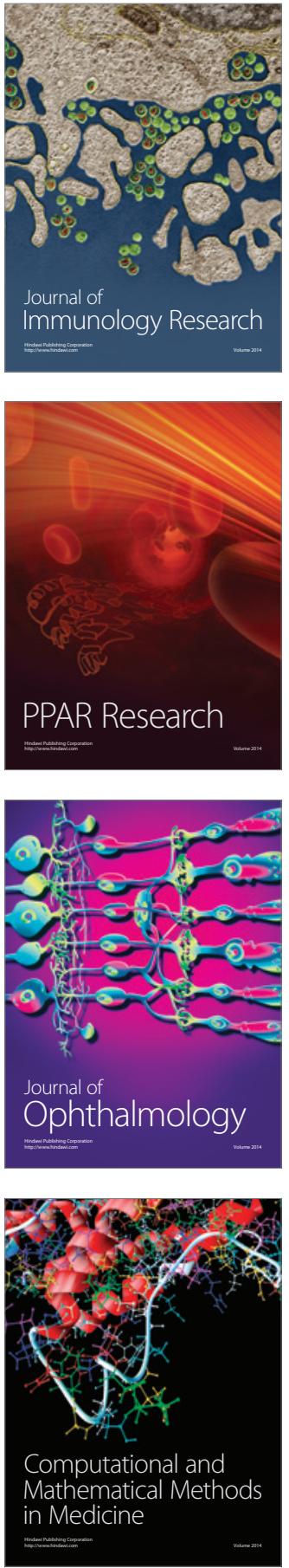

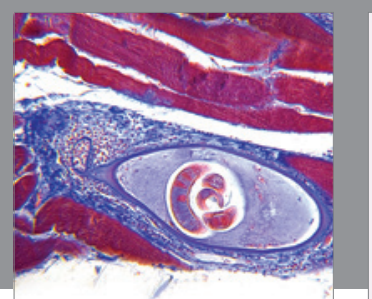

Gastroenterology Research and Practice

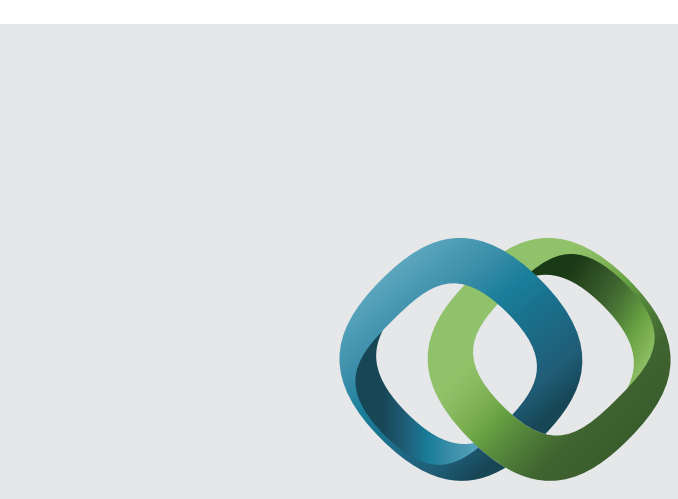

\section{Hindawi}

Submit your manuscripts at

http://www.hindawi.com
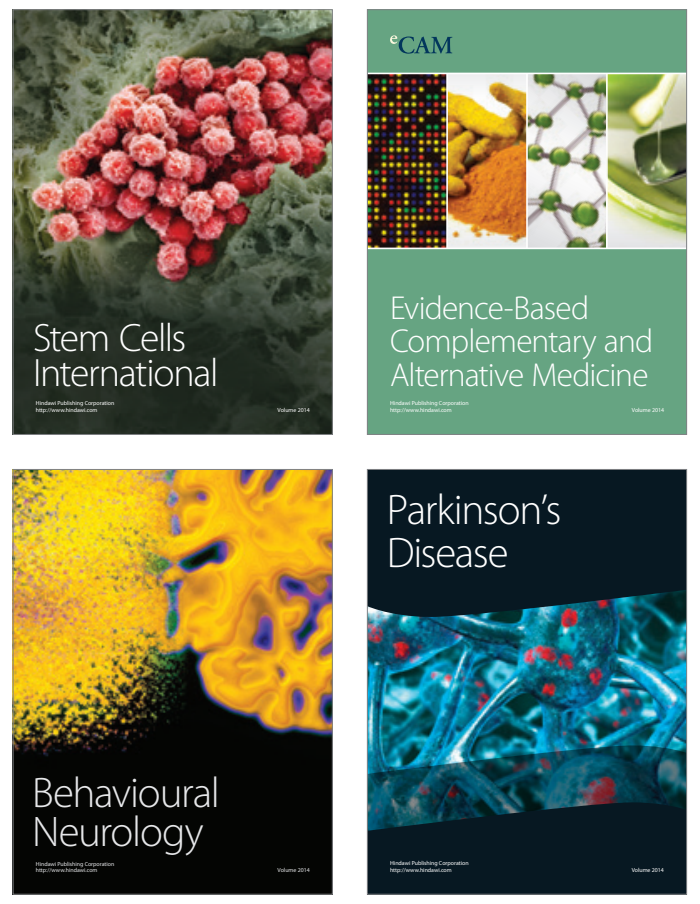
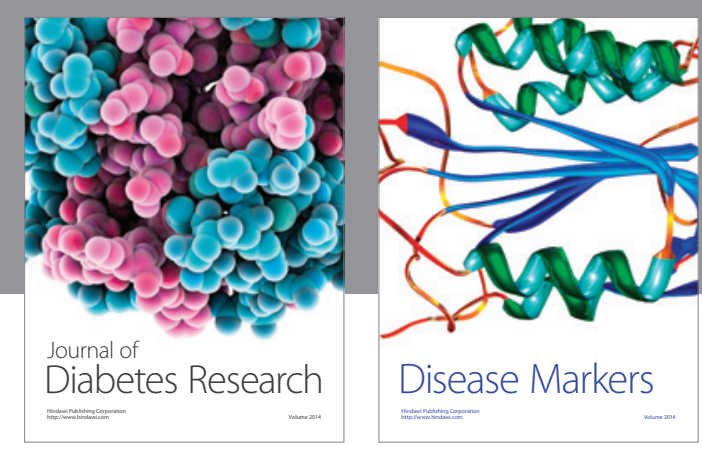

Disease Markers
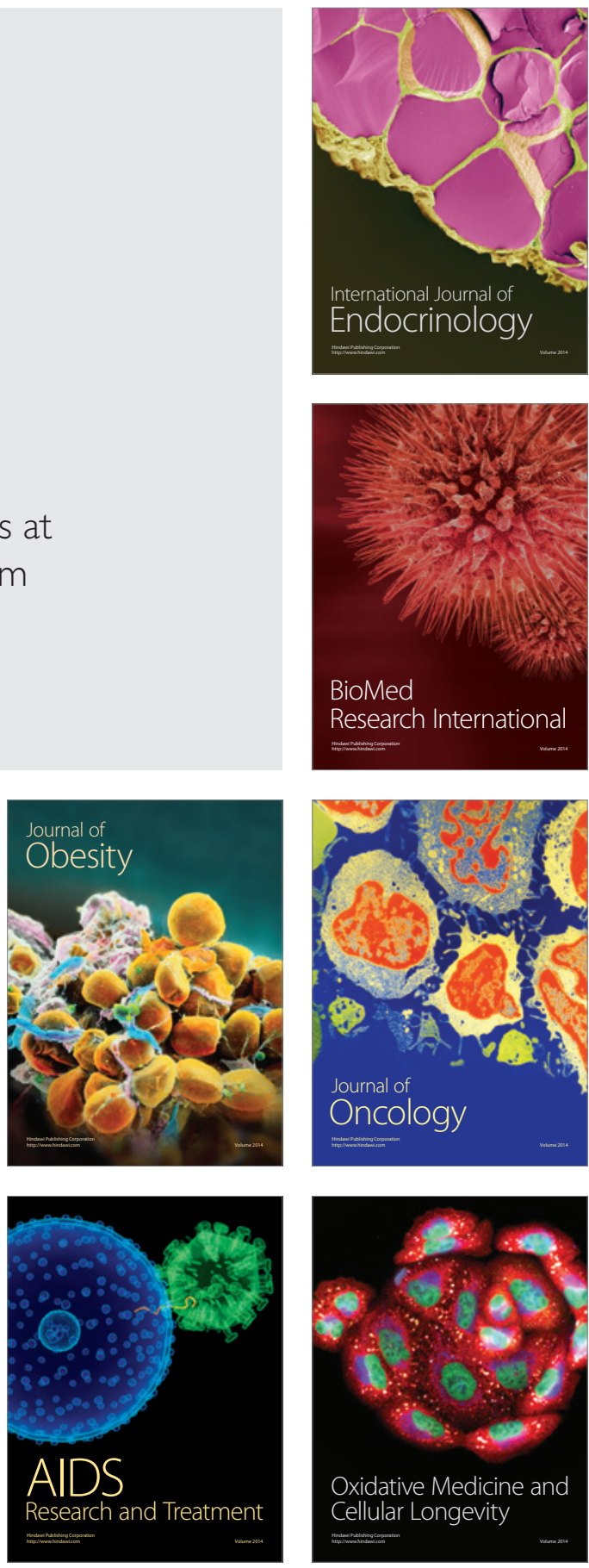